\title{
On hidden Markov chains and finite stochastic systems Peter Spreij円
}

November 8, 2018

\begin{abstract}
In this paper we study various properties of finite stochastic systems or hidden Markov chains as they are alternatively called. We discuss their construction following different approaches and we also derive recursive filtering formulas for the different systems that we consider. The key tool is a simple lemma on conditional expectations.
\end{abstract}

Keywords: Markov Chain, Hidden Markov Chain, Recursive Filtering, Stochastic System.

Mathematics Subject Classification: 60G42, 60J10, 93E11

\footnotetext{
${ }^{1}$ Korteweg-de Vries Institute for Mathematics, Universiteit van Amsterdam, Plantage Muidergracht 24, 1018 TV Amsterdam
} 



\section{Introduction}

In this paper we consider Hidden Markov Chains (probabilistic functions of a Markov chain) that, like the underlying Markov chain, take on finitely many values. The observed process is denoted by $Y$, the underlying chain by $X$. Hidden Markov chains are such that probabilities of future events of $X$ and $Y$ given the past only depend on the current state of $X$. Typically this means that $X$ satisfies the role of a state process as it is used in stochastic system theory. One of the aims of the present paper is to shed some more light on the relation between stochastic systems and hidden Markov chains. There are two slightly different definitions of stochastic systems, related by a time shift of the observed process. We will see that a hidden Markov chain satisfies both relations. We will also discuss various constructions of a hidden Markov chain. These constructions allow different factorization and splitting properties of conditional probabilities of the bivariate process $(X, Y)$. We will also study for the different constructions the filtering and prediction problems and show that the solutions coincide if one deals with a hidden Markov chain in the way we define it. The paper is organized as follows.

In section 2 we describe the probabilistic behaviour of the joint process $(X, Y)$ in more detail using the outer product of $X$ and $Y$ and by using properties of Kronecker products of matrices.

In section 3 we present a somewhat different look at hidden Markov chains. It is shown that certain necessary properties of a hidden Markov chain are actually sufficient to construct one. The convenient tool is a simple lemma, that is presented in the appendix, on conditional expectations that involves a finitely generated $\sigma$-algebra. It is also shown that hidden Markov chains are nothing else but what in the engineering literature are called stochastic systems. In particular it is shown that hidden Markov chains satisfy two different notions of stochastic systems. It is also shown how these two notions are interrelated. This is done in section 1 .

In section 5 we show how various filtering and prediction formulas are simple consequences of the key lemma on conditional expectations of the appendix.

\section{Preliminaries}

Let $(\Omega, \mathcal{F}, P)$ be a probability space on which all the random variables to be encountered below are defined. Consider the following model for what we will call later a Hidden Markov Chain (HMC).

$$
\begin{aligned}
X_{t} & =A X_{t-1}+\varepsilon_{t}, X_{0} \\
Y_{t} & =H_{t} X_{t}
\end{aligned}
$$

Here the state process $X$ is modelled as a Markov process on the set $E=$ $\left\{e_{1}, \ldots, e_{n}\right\}$ of basis vectors of $\mathbb{R}^{n}$. Moreover, this process is supposed to be time-homogeneous with $A$ the matrix of one step transitions probabilities: $A_{i j}=P\left(X_{t+1}=e_{i} \mid X_{t}=e_{j}\right)$. The process $\left\{\varepsilon_{t}\right\}$ is then a martingale difference 
sequence adapted to the filtration generated by $X$, see [2, page 17]. Throughout the paper we assume that each state $e_{i}$ is visited at least once by $X$. If this were not the case, this can always be accomplished by reducing the state space of $X$ by taking basis vectors of a lower dimensional Euclidean space.

The observation or output process $Y$ takes its values in the set $F=\left\{f_{1}, \ldots, f_{m}\right\}$ of basis vectors of $\mathbb{R}^{m}$. The matrices $\left\{H_{t}\right\}$ are assumed to form an iid sequence, independent of $\left\{X_{t}\right\}$, and each column of any of these matrices is assumed to be a random element of $F$. Clearly each $H_{t}$ is the incidence matrix of a random map from $E$ in $F$. Indeed, if $Y_{t}=h_{t}\left(X_{t}\right)$, with the $h_{t}$ random maps from $E$ into $F$, then we can write $Y_{t}=\sum_{i=1}^{n} h_{t}\left(e_{i}\right) 1_{\left\{X_{t}=e_{i}\right\}}$. So we define $H_{t}=\left[h_{t}\left(e_{1}\right), \ldots, h_{t}\left(e_{n}\right)\right]$ to get (2) $)$.

We will only need the distributions of the colums of $H_{t}$ (equivalently, the marginal distributions of the $h_{t}\left(e_{i}\right)$. These are specified by the expectation $E H_{t}=G$. We assume (without loss of generality) the non-degeneracy condition that none of the rows of $G$ is zero.

Define the filtration $\mathbb{F}=\left\{\mathcal{F}_{t}\right\}$ by $\mathcal{F}_{t}=\sigma\left\{X_{0}, \ldots, X_{t}, H_{0}, \ldots, H_{t}\right\}$. Clearly both $X$ and $Y$ are adapted to this filtration, and so is the sequence $\left\{\varepsilon_{t}\right\}$ which is even a martingale difference sequence w.r.t $\mathbb{F}$, because of the independence of the sequences $\left\{X_{t}\right\}$ and $\left\{H_{t}\right\}$.

In the current set up, also the joint process $\left\{\left(X_{t}, Y_{t}\right)\right\}$ is Markov. For completeness we give its transition probabilities, already given in [1], and derive these using simple properties of conditional expectations.

Proposition 2.1 The joint process $\left\{\left(X_{t}, Y_{t}\right)\right\}$ is Markov with respect to $\mathbb{F}$ and the conditional transition probabilities are given by

$$
P\left(X_{t}=e_{i}, Y_{t}=f_{j} \mid \mathcal{F}_{t-1}\right)=e_{i}^{\top} \operatorname{diag}\left(A X_{t-1}\right) G^{\top} f_{j}
$$

Proof. Notice first that the indicator of the event $\left\{X_{t}=e_{i}, Y_{t}=f_{j}\right\}$ equals $e_{i}^{\top} X_{t} Y_{t}^{\top} f_{j}$. Hence we can rewrite the conditional probability in equation (3) as $E\left[e_{i}^{\top} X_{t} Y_{t}^{\top} f_{j} \mid \mathcal{F}_{t-1}\right]$. So we compute

$$
\begin{aligned}
E\left[X_{t} Y_{t}^{\top} \mid \mathcal{F}_{t-1}\right] & =E\left[X_{t} X_{t}^{\top} H_{t}^{\top} \mid \mathcal{F}_{t-1}\right] \\
& =E\left[E\left[X_{t} X_{t}^{\top} H_{t}^{\top} \mid \mathcal{F}_{t-1} \vee \sigma\left(H_{t}\right)\right] \mid \mathcal{F}_{t-1}\right] \\
& =E\left[E\left[X_{t} X_{t}^{\top} \mid \mathcal{F}_{t-1} \vee \sigma\left(H_{t}\right)\right] H_{t}^{\top} \mid \mathcal{F}_{t-1}\right] \\
& =E\left[E\left[\operatorname{diag}\left(X_{t}\right) \mid \mathcal{F}_{t-1} \vee \sigma\left(H_{t}\right)\right] H_{t}^{\top} \mid \mathcal{F}_{t-1}\right] \\
& =E\left[\operatorname{diag}\left(A X_{t-1}\right) H_{t}^{\top} \mid \mathcal{F}_{t-1}\right] \\
& =\operatorname{diag}\left(A X_{t-1}\right) E\left[H_{t}^{\top} \mid \mathcal{F}_{t-1}\right] \\
& =\operatorname{diag}\left(A X_{t-1}\right) G^{\top}
\end{aligned}
$$

The result follows.

We will see in section 1 that it follows from proposition 2.1 that the pair $(X, Y)$ forms a stochastic system in the sense of [5].

We continue with giving an alternative expression for the matrix of one step 
transition probabilities of the joint chain $(X, Y)$. The state space of this chain consists of all the $n m$ pairs $\left(e_{i}, f_{j}\right)$. These are renamed and ordered as follows: $s_{(j-1) n+i}=\left(e_{i}, f_{j}\right)$ for $i \in\{1, \ldots, n\}$ and $j \in\{1, \ldots, m\}$. Clearly the map $(i, j) \mapsto(i-1) m+j$ is bijective from $\{1, \ldots, n\} \times\{1, \ldots, m\}$ onto $\{1, \ldots, n m\}$. Instead of working with $(X, Y)$ we will use the chain $Z$ that carries the same information and which is defined by $Z_{t}=\operatorname{vec}\left(X_{t} Y_{t}^{\top}\right)$. Recall that the vecoperator applied to a matrix results in a vector where all the columns of this matrix are stacked one underneath the other [4, p. 30]. Then clearly the state space of $Z$ is the set of basis vectors of $\mathbb{R}^{n m}$. If we call this set $\left\{z_{1}, \ldots, z_{n m}\right\}$ we see that $\left(X_{t}, Y_{t}\right)=s_{k}$ iff $Z_{t}=z_{k}$. Notice also the following relations. $Z_{t}=Y_{t} \otimes X_{t}, X_{t}=\left(\mathbf{1}_{m}^{\top} \otimes I_{n}\right) Z_{t}$ and $Y_{t}=\left(I_{m} \otimes \mathbf{1}_{n}^{\top}\right) Z_{t}$. Here $I_{m}$ is the $m$ dimensional identity matrix and $\mathbf{1}_{n}$ is the $n$-dimensional column vector with all its elements equal to one.

According to proposition 2.1 we now get that the $n m \times n m$ matrix $Q$ of transition probabilities of $Z$ can be decomposed as a matrix with $m^{2}$ blocks $Q_{i j}$ that are equal to $\operatorname{diag}\left(G_{i}\right) A$, where $G_{i}$. is the $i$-th row of $G$. For a more compact formulation we introduce (like in [7]) the following notation. Let $\Delta(G)$ be the $n m \times n$ matrix defined by

$$
\Delta(G)=\left[\begin{array}{c}
\operatorname{diag}\left(G_{1 .}\right) \\
\vdots \\
\operatorname{diag}\left(G_{m .}\right)
\end{array}\right]
$$

Using the notation $\Delta(G)$ we can now write

$$
Q=\Delta(G) A\left(\mathbf{1}_{m}^{\top} \otimes I_{n}\right)
$$

In the next lemma we gather some computational results for the $\Delta$-operator, that might be of independent interest. Other properties are described in [7].

Lemma 2.2 For any matrices $G \in \mathbb{R}^{m \times n}, M \in \mathbb{R}^{p \times m}$ and $N \in \mathbb{R}^{p \times n}$ and for any vectors $w \in \mathbb{R}^{n}, v \in \mathbb{R}^{m}$ we have

$$
\begin{aligned}
M G & =\left(M \otimes \mathbf{1}_{n}^{\top}\right) \Delta(G) \\
\left(I_{m} \otimes \operatorname{diag}(w)\right) \operatorname{vec}\left(G^{\top}\right) & =\Delta(G) w \\
\operatorname{vec}\left(\operatorname{diag}(w) G^{\top}\right) & =\Delta(G) w
\end{aligned}
$$

Proof. By direct calculation.

The expression (4) for $Q$ can also be obtained through simple matrix manipulations and by application of lemma 2.2. By definition of $Q$ we have 
$E\left[Z_{t+1} \mid \mathcal{F}_{t}\right]=Q Z_{t}$. So we compute the conditional expectation

$$
\begin{aligned}
E\left[Z_{t+1} \mid \mathcal{F}_{t}\right] & =E\left[\operatorname{vec}\left(X_{t+1} Y_{t+1}^{\top}\right) \mid \mathcal{F}_{t}\right] \\
& =\operatorname{vec}\left(E\left[X_{t+1} Y_{t+1}^{\top} \mid \mathcal{F}_{t}\right]\right) \\
& =\operatorname{vec}\left(E\left[X_{t+1} X_{t+1}^{\top} H_{t+1}^{\top} \mid \mathcal{F}_{t}\right]\right) \\
& =\operatorname{vec}\left(\operatorname{diag}\left(A X_{t}\right) G^{\top}\right) \\
& =\left(I_{m} \otimes \operatorname{diag}\left(A X_{t}\right)\right) \operatorname{vec}\left(G^{\top}\right) \\
& =\Delta(G) A X_{t} \\
& =\Delta(G) A\left(\mathbf{1}_{m}^{\top} \otimes I_{n}\right) Z_{t} \\
& =Q Z_{t}
\end{aligned}
$$

Here we used in the fifth equality a known result for the vec-operator of the product of three matrices (see [ [ tion (6).

If the vector $p_{0}$ represents the initial distribution of $X$, then the initial distribution of $Z$ is given by the vector $E Z_{0}=\operatorname{vec}\left(\operatorname{diag}\left(p_{0}\right) G^{\top}\right): E Z_{0}=E \operatorname{vec}\left(X_{0} Y_{0}^{\top}\right)=$ $\operatorname{vec}\left(E \operatorname{diag}\left(X_{0}\right) H_{0}^{\top}\right)=\operatorname{vec}\left(\operatorname{diag}\left(p_{0}\right) G^{\top}\right)$, since $X_{0}$ and $H_{0}$ are independent. No-

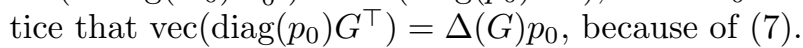

Similarly one can show that $\Delta(G) \pi$ is an invariant probability vector for $Z$, if $X$ has an invariant probability vector $\pi$.

It is easy to see from equation (3) that the "factorization property" [3] holds:

$$
P\left(X_{t}=e_{i}, Y_{t}=f_{j} \mid \mathcal{F}_{t-1}\right)=P\left(Y_{t}=f_{j} \mid X_{t}=e_{i}\right) P\left(X_{t}=e_{i} \mid X_{t-1}\right)
$$

The proof is straight forward from proposition 2.1 (used in the first equality below):

$$
\begin{aligned}
P\left(X_{t}=e_{i}, Y_{t}=f_{j} \mid \mathcal{F}_{t-1}\right) & =e_{i}^{\top} \operatorname{diag}\left(A X_{t-1}\right) G^{\top} f_{j} \\
& =\left(A X_{t-1}\right)^{\top} \operatorname{diag}\left(e_{i}\right) G^{\top} f_{j} \\
& =\left(A X_{t-1}\right)^{\top} e_{i} e_{i}^{\top} G^{\top} f_{j} \\
& =P\left(X_{t}=e_{i} \mid X_{t-1}\right) G_{j i} \\
& =P\left(X_{t}=e_{i} \mid X_{t-1}\right) P\left(Y_{t}=f_{j} \mid X_{t}=e_{i}\right) .
\end{aligned}
$$

Using the matrix $\Delta(G)$ and lemma 2.2 we can also compactly rephrase the factorization property (8). It becomes

$$
E\left[Z_{t} \mid \mathcal{F}_{t-1}\right]=\Delta(G) E\left[X_{t} \mid X_{t-1}\right], \forall t .
$$

This can be verified as follows. First, using proposition 2.1 again, we rewrite (8) as

$$
e_{i}^{\top} E\left[X_{t} Y_{t}^{\top} \mid \mathcal{F}_{t-1}\right] f_{j}=G_{j i} e_{i}^{\top} E\left[X_{t} \mid \mathcal{F}_{t-1}\right] .
$$

Since the right hand side of this equality equals $f_{j}^{\top} G \operatorname{diag}\left(e_{i}\right) E\left[X_{t} \mid \mathcal{F}_{t-1}\right]$, which is equal to $f_{j}^{\top} G \operatorname{diag}\left(E\left[X_{t} \mid \mathcal{F}_{t-1}\right]\right) e_{i}$, we get

$$
E\left[X_{t} Y_{t}^{\top} \mid \mathcal{F}_{t-1}\right]=\operatorname{diag}\left(E\left[X_{t} \mid X_{t-1}\right]\right) G^{\top} .
$$


Since $\operatorname{vec}\left(X_{t} Y_{t}^{\top}\right)=Z_{t}$ and

$$
\operatorname{vec}\left(\operatorname{diag}\left(E\left[X_{t} \mid X_{t-1}\right]\right) G^{\top}\right)=\left(I_{m} \otimes \operatorname{diag}\left(E\left[X_{t} \mid X_{t-1}\right]\right)\right) \operatorname{vec}\left(G^{\top}\right),
$$

we use (6) to write the RHS of this last equality as $\Delta(G) E\left[X_{t} \mid X_{t-1}\right]$, from which (9) follows.

Remark 2.3 The validity of equation (9) has been seen to be a consequence of the special form of the transition matrix $Q$ in (4). But also the converse holds. If (9) holds, we get at once that $Z$ is $\mathbb{F}$-Markov, if $X$ is $\mathbb{F}$-Markov. And if we denote the transition matrix of $Z$ by $Q$ and that of $X$ by $A$, we automatically get (4) back. See proposition 3.1.

As an alternative to looking at the bivariate process $(X, Y)$ via the process $Z$ as above, we study the process $W$, again built from $X$ and $Y$ and defined by $W_{t}=Y_{t-1} \otimes X_{t}$ for $t \geq 1$. Along with this process we consider the filtration $\mathbb{G}$ of $\sigma$-algebras $\mathcal{G}_{t}:=\sigma\left\{H_{0}, \ldots, H_{t-1}, X_{0}, \ldots, X_{t}\right\}$. Then $W$ is $\mathbb{G}$-adapted and the $\mathcal{G}_{t}$ and the $\mathcal{F}_{t}$ are related by $\mathcal{F}_{t-1} \vee \sigma\left(X_{t}\right)=\mathcal{G}_{t}$ and $\mathcal{G}_{t} \vee \sigma\left(H_{t}\right)=\mathcal{F}_{t}$.

Then by similar computations as we carried out before and by using the Markov property of $Z$ we obtain the relations

$$
\begin{aligned}
E\left[W_{t} \mid \mathcal{F}_{t-1}\right] & =\left(I_{m} \otimes A\right) Z_{t-1}, \\
E\left[W_{t} \mid \mathcal{G}_{t-1}\right] & =G X_{t-1} \otimes A X_{t-1}=\left(I_{m} \otimes A\right) \Delta(G) X_{t-1} .
\end{aligned}
$$

In particular it follows that $W$ is $\mathbb{G}$-Markov (and hence the pair $(X, Y)$ is a stochastic system in the sense of [6], see section (1) with transition matrix

$$
R:=\left(I_{m} \otimes A\right) \Delta(G)\left(\mathbf{1}_{m}^{\top} \otimes I_{n}\right) .
$$

Observe also that $W$ has the splitting property

$$
E\left[W_{t+1} \mid \mathcal{G}_{t}\right]=E\left[Y_{t} \mid \mathcal{G}_{t}\right] \otimes E\left[X_{t+1} \mid \mathcal{G}_{t}\right],
$$

which immediately follows from (11).

Remark 2.4 The assumption in this section that the sequence $\left\{H_{t}\right\}$ is iid with $E H_{t}=G$ can in principle be relaxed to assuming that $\left\{H_{t}-G\right\}$ is a martingale difference sequence with respect to its own filtration without changing the results of this section. However, this only appears to be a relaxation, in fact they are equivalent assumptions. Indeed, let $\left\{H_{t}-G\right\}$ be a martingale difference sequence and consider $k_{t}=\operatorname{vec}\left(H_{t}\right)$. Then $k_{t}$ takes its values in the set of basis vectors of $\mathbb{R}^{m n}$ and $\left\{k_{t}-\operatorname{vec}(G)\right\}$ is again a martingale difference sequence. Let $e$ be one of these basis vectors. Then $P\left(k_{t+1}=e \mid k_{0}, \ldots, k_{t}\right)=e^{\top} E\left[k_{t+1} \mid k_{0}, \ldots, k_{t}\right]=$ $e^{\top} \operatorname{vec}(G)$, which doesn't depend on $k_{0}, \ldots, k_{t}$, nor on time. Hence $\left\{k_{t}\right\}$ is an iid sequence and so is $\left\{H_{t}\right\}$.

We can also replace (2) with the equivalent equation

$$
Y_{t}=G X_{t}+\eta_{t}
$$


where $\eta$ forms a martingale difference sequence with respect to $\left\{\mathcal{F}_{t}\right\}$, and it even holds that $\eta_{t}=Y_{t}-E\left[Y_{t} \mid \sigma\left(X_{t}\right) \vee \mathcal{F}_{t-1}\right]=Y_{t}-E\left[Y_{t} \mid \mathcal{G}_{t}\right]$. The combined set of equations (11) and (14) are of the form that is commonly used in (stochastic) systems theory. We will come back to stochastic systems in section 1 .

Remark 2.5 As a final remark we notice that all the properties mentioned above in terms of conditional expectations given the $\sigma$-algebras $\mathcal{F}_{t}$ and $\mathcal{G}_{t}$ remain valid if we replace the former one with $\sigma\left\{X_{0}, \ldots, X_{t}, Y_{0}, \ldots, Y_{t}\right\}$ and the latter one with $\sigma\left\{X_{0}, \ldots, X_{t}, Y_{0}, \ldots, Y_{t-1}\right\}$. Hence the law of the bivariate process $(X, Y)$, being a Markov chain with respect to its own filtration, is completely specified by the matrices $A$ and $G$ and the initial law of $X$. It follows that any bivariate Markov process $(X, Y)$, that is such that the transition matrix $Q$ of the associated process $Z=Y \otimes X$ is of the form (任) and that has initial law $E Z_{0}=\Delta(G) p_{0}$ where $p_{0}=E X_{0}$, can be constructed as the output of the system (11) and (2).

In view of remark 2.5 above we adopt the following

Definition 2.6 A bivariate process $(X, Y)$ that assumes finitely many values is called a Hidden Markov Chain (HMC) if the process $Z=Y \otimes X$ is Markov with respect to the filtration $\mathbb{F}=\left\{\mathcal{F}_{t}\right\}$ defined by $\mathcal{F}_{t}=\sigma\left\{X_{0}, \ldots, X_{t}, Y_{0}, \ldots, Y_{t}\right\}$ and if its matrix of transition probabilities is given by (4).

\section{Alternative descriptions of a HMC}

There are various ways to describe some properties of a stochastic system or a Hidden Markov chain. We mention a few possibilities and show how these can be used as building stones for a HMC.

Let $X$ and $Y$ be two stochastic processes taking values in the sets $E$ and $F$ respectively, like in section 2. Let $Z$ again be the process $Y \otimes X$. For the time being no further assumptions on $X$ and $Y$ are imposed, except that redundant states are excluded in the sense that each state of $X$ is visited at least once with probability one and likewise for $Y$.

In this section (and all subsequent ones) we assume that for all $t$ the $\sigma$-algebra $\mathcal{F}_{t}$ is generated by $X_{0}, \ldots, X_{t}, Y_{0}, \ldots, Y_{t}$. The family $\left\{\mathcal{F}_{t}\right\}$ is again denoted by $\mathbb{F}$. We also consider the process $W$ again, with $W_{t}=Y_{t-1} \otimes X_{t}$, adapted to the filtration $\mathbb{G}=\left\{\mathcal{G}_{t}\right\}$, with $\mathcal{G}_{t}$ generated by $X_{0}, \ldots, X_{t}, Y_{0}, \ldots, Y_{t-1}$. Notice again the relations

$$
\begin{aligned}
\mathcal{F}_{t} & =\mathcal{G}_{t} \vee \sigma\left(Y_{t}\right) \\
\mathcal{G}_{t} & =\mathcal{F}_{t-1} \vee \sigma\left(X_{t}\right)
\end{aligned}
$$

\subsection{Alternative description of $\mathbf{Z}$}

We now list a set possible properties that the processes $X, Y$ and $Z$ may possess. 
1. The process $Z$ is time homogeneous $\mathbb{F}$-Markov with matrix $Q$ of transition probabilities, so $E\left[Z_{t+1} \mid \mathcal{F}_{t}\right]=Q Z_{t}$. Moreover we assume that this conditional expectation only depends on $X_{t}$, which implies that there exists a matrix $\bar{Q}$ such that $E\left[Z_{t+1} \mid \mathcal{F}_{t}\right]=\bar{Q} X_{t}=\bar{Q}\left(\mathbf{1}_{m}^{\top} \otimes I_{n}\right) Z_{t}, \forall t$. Hence $Q=\bar{Q}\left(\mathbf{1}_{m}^{\top} \otimes I_{n}\right)$.

2. The output property holds:

$$
E\left[Y_{t} \mid \mathcal{G}_{t}\right]=E\left[Y_{t} \mid \mathcal{F}_{t-1} \vee \sigma\left(X_{t}\right)\right]=E\left[Y_{t} \mid \sigma\left(X_{t}\right)\right], \forall t .
$$

If this property holds, we use the matrix $G$ defined by $E\left[Y_{t} \mid \sigma\left(X_{t}\right)\right]=G X_{t}$, where we also assume that $G$ is not depending on $t$. $G$ is then such that the columns $G_{\cdot i}$ are equal to $E\left[Y_{t} \mid X_{t}=e_{i}\right]$.

3. The extended output property holds:

$$
E\left[Z_{t} \mid \mathcal{G}_{t}\right]=E\left[Z_{t} \mid \mathcal{F}_{t-1} \vee \sigma\left(X_{t}\right)\right]=E\left[Z_{t} \mid \sigma\left(X_{t}\right)\right], \forall t .
$$

In this case we define the matrix $B$ (assumed to be independent of $t$ ) by $E\left[Z_{t} \mid \sigma\left(X_{t}\right)\right]=B X_{t}$.

4. The factorization property holds: There exists a matrix $K \in \mathbb{R}^{m \times n}$ such that

$$
E\left[Z_{t} \mid \mathcal{F}_{t-1}\right]=\Delta(K) E\left[X_{t} \mid \mathcal{F}_{t-1}\right], \forall t
$$

First we comment on the factorization property. We showed that it is valid for the HMC of section 2. But one can always factorize $E\left[Z_{t} \mid \mathcal{F}_{t-1}\right]$ with a second factor $E\left[X_{t} \mid \mathcal{F}_{t-1}\right]$ as in $(16)$, however in general the left factor is a random $\left(\mathcal{F}_{t-1}\right.$-measurable) diagonal matrix, see equation (19) below.

Denote by $P_{i}$ the conditional measure on $(\Omega, \mathcal{F})$ given $X_{t}=e_{i}$. Expectation with respect to these measures will be denoted by $E_{i}$, with the understanding that expectations $E_{i} U$ are set equal to zero, if $P\left(X_{t}=e_{i}\right)=0$ (cf. the appendix). Then for any sub- $\sigma$-algebra $\mathcal{F}^{0}$ of $\mathcal{F}$ and any integrable random variable $U$ we have from equation (37) in the appendix the relation

$$
E\left[U 1_{\left\{X_{t}=e_{i}\right\}} \mid \mathcal{F}^{0}\right]=E_{i}\left[U \mid \mathcal{F}^{0}\right] P\left(X_{t}=e_{i} \mid \mathcal{F}^{0}\right) .
$$

Application of equation (17) with $U=Y_{t}^{\top}, \mathcal{F}^{0}=\mathcal{F}_{t-1}$ for all $i$ yields

$$
E\left[X_{t} Y_{t}^{\top} \mid \mathcal{F}_{t-1}\right]=\operatorname{diag}\left(E\left[X_{t} \mid \mathcal{F}_{t-1}\right]\right) E^{\top},
$$

where $E^{\top}$ is the transpose of the matrix $E$ that has columns $E_{i}\left[Y_{t} \mid \mathcal{F}_{t-1}\right]$. Apply then (7) to get

$$
E\left[Z_{t} \mid \mathcal{F}_{t-1}\right]=\Delta(E) E\left[X_{t} \mid \mathcal{F}_{t-1}\right] .
$$

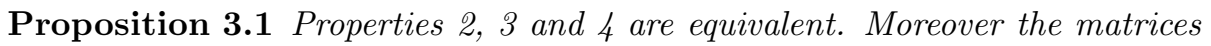
$B, G$ and $K$ are related via $B=\Delta(G)$ and $K=G$. 
Proof. Trivially the output property 2 follows from the extended output property 3 by left multiplication with $I_{m} \otimes \mathbf{1}_{n}^{\top}$.

Conversely, assume that the output property holds. Then we have $E\left[Z_{t} \mid \mathcal{F}_{t-1} \vee\right.$ $\left.\sigma\left(X_{t}\right)\right]=E\left[Y_{t} \mid \mathcal{F}_{t-1} \vee \sigma\left(X_{t}\right)\right] \otimes X_{t}=E\left[Y_{t} \mid X_{t}\right] \otimes X_{t}=E\left[Z_{t} \mid X_{t}\right]$, which shows that the extended output property holds.

To see the relation between $B$ and $G$, notice that in this case we have $B X_{t}=$ $E\left[Z_{t} \mid X_{t}\right]=E\left[Y_{t} \mid X_{t}\right] \otimes X_{t}=G X_{t} \otimes X_{t}=\operatorname{vec}\left(X_{t} X_{t}^{\top} G^{\top}\right)=\operatorname{vec}\left(\operatorname{diag}\left(X_{t}\right) G^{\top}\right)=$ $\Delta(G) X_{t}$. Here we used the usual relations between the vec-operator and Kronecker products as well as (7) in the last equality.

Assume that the extended output property holds. Use then reconditioning in (15) to get: $E\left[Z_{t} \mid \mathcal{F}_{t-1}\right]=E\left[E\left[Z_{t} \mid \mathcal{F}_{t-1} \vee \sigma\left(X_{t}\right)\right] \mid \mathcal{F}_{t-1}\right]=E\left[E\left[Z_{t} \mid X_{t}\right] \mid \mathcal{F}_{t-1}\right]=$ $B E\left[X_{t} \mid \mathcal{F}_{t-1}\right]$. It follows from (19) that $B=\Delta(E)$, but since $B$ is nonrandom, the validity of the factorization property follows.

Conversely, assume that the factorization property 1 holds. Take expectations in (16). Then $E Z_{t}=\Delta(K) E X_{t}$. From the definition of $G$ (in property 2) we get $\left.E Z_{t}=E E\left[Y_{t} \mid \sigma\left(X_{t}\right)\right] \otimes X_{t}\right]=E\left(G X_{t} \otimes X_{t}\right)=\Delta(G) E X_{t}$. Since for each $i$ there is a $t$ such that the $i$-th component of $E X_{t}$ is strictly positive, it follows from the blockwise diagonal structure of the $\Delta$-matrices that $\Delta(G)=\Delta(K)$ and $G=K$.

Next we show that the output property holds. Assume for a moment that all elements of $E X_{t}$ are positive. According to equations (35) and (33) we have

$$
E\left[Y_{t} \mid \mathcal{F}_{t-1} \vee \sigma\left(X_{t}\right)\right]=\sum_{i} \frac{E\left[Y_{t} e_{i}^{\top} X_{t} \mid \mathcal{F}_{t-1}\right]}{e_{i}^{\top} E\left[X_{t} \mid \mathcal{F}_{t-1}\right]} e_{i}^{\top} X_{t}
$$

Since $Y_{t} e_{i}^{\top} X_{t}=\left(I_{m} \otimes e_{i}^{\top}\right) Z_{t}$ and using the factorization property, we can rewrite this as

$$
\sum_{i} \frac{\left(I_{m} \otimes e_{i}^{\top}\right) \Delta(G) E\left[X_{t} \mid \mathcal{F}_{t-1}\right]}{e_{i}^{\top} E\left[X_{t} \mid \mathcal{F}_{t-1}\right]} e_{i}^{\top} X_{t} .
$$

Because $\left(I_{m} \otimes e_{i}^{\top}\right) \Delta(G)=G e_{i} e_{i}^{\top}$, this reduces to

$$
G \sum_{i} \frac{e_{i} e_{i}^{\top} E\left[X_{t} \mid \mathcal{F}_{t-1}\right]}{e_{i}^{\top} E\left[X_{t} \mid \mathcal{F}_{t-1}\right]} e_{i}^{\top} X_{t}
$$

which in turn is nothing else but $G X_{t}$, from which we obtain the output property. In the case where the vector $E X_{t}$ has some elements equal to zero, the above procedure is still valid, provided we let the summation indices run through the set $\left\{i: e_{i}^{\top} E X_{t}>0\right\}$.

Similar to what we found in the previous section we have

Proposition 3.2 Assume that the factorization property 4 holds (or, equivalently in view of proposition 3.1, the output or extended output property). Then the following two statements are equivalent.

(i) $Z$ is $\mathbb{F}$-Markov with transition matrix $Q=\bar{Q}\left(\mathbf{1}_{m}^{\top} \otimes I_{n}\right)$.

(ii) $X$ is $\mathbb{F}$-Markov with transition matrix $A$

Furthermore we have in each of these situations the relation $\bar{Q}=\Delta(G) A$. 
Proof. (i) $\Rightarrow$ (ii): Clearly $X$ is $\mathbb{F}$-Markov with transition matrix $A=\left(\mathbf{1}_{m}^{\top} \otimes I_{n}\right) \bar{Q}$ and then it follows from the factorization property that $\bar{Q} X_{t-1}=E\left[Z_{t} \mid \mathcal{F}_{t-1}\right]=$ $\Delta(G) E\left[X_{t} \mid \mathcal{F}_{t-1}\right]=\Delta(G) A X_{t-1}$.

Conversely, (ii) $\Rightarrow$ (i) follows in a similar way. $E\left[Z_{t} \mid \mathcal{F}_{t-1}\right]=\Delta(G) E\left[X_{t} \mid \mathcal{F}_{t-1}\right]=$ $\Delta(G) A X_{t-1}=\Delta(G) A\left(\mathbf{1}_{m}^{\top} \otimes I_{n}\right) Z_{t-1}$, so $Z$ is $\mathbb{F}$-Markov with transition matrix $Q=\Delta(G) A\left(\mathbf{1}_{m}^{\top} \otimes I_{n}\right)$.

Remark 3.3 The main implication of proposition 3.2 is that the proces $Z$ is a Markov chain whose transition probabilities only depend on the past value of $X$, if one starts out with a $\mathbb{F}$-Markov chain $X$ and imposes that the output condition holds. Clearly, if $X$ is just Markov with respect to its own filtration and if the factorization property is replaced with the stronger condition $E\left[Z_{t} \mid \mathcal{F}_{t-1}\right]=$ $\Delta(K) E\left[X_{t} \mid \mathcal{F}_{t-1}\right]$, the same conclusion follows.

Remark 3.4 We also observe, like in section 2, that the fact that $Z$ is $\mathbb{F}$ Markov with $\bar{Q}=\Delta(G) A$ implies that $W$ is $\mathbb{G}$-Markov, with transition matrix $R=\left(I_{m} \otimes A\right) \Delta(G)$. One easily checks that with the present choice of the filtrations equations (10) and (11) remain valid, and that in particular the factorization property holds.

\subsection{Alternative description of $\mathrm{W}$}

Like in subsection 3.2, we can also list a set of desirable properties of $W$. Consider thereto

1. $W$ is a time homogeneous $\mathbb{G}$-Markov chain with a transition matrix $R$. Moreover, we have that conditional expectation $E\left[W_{t+1} \mid \mathcal{G}_{t}\right]$ depends only on $X_{t}$. This means that there is a matrix $\bar{R}$ such that $R=\bar{R}\left(\mathbf{1}_{m}^{\top} \otimes I_{n}\right)$.

2. The splitting property holds:

$$
E\left[W_{t} \mid \mathcal{G}_{t-1}\right]=E\left[Y_{t-1} \mid \mathcal{G}_{t-1}\right] \otimes E\left[X_{t} \mid \mathcal{G}_{t-1}\right], \forall t .
$$

Then we have similar to proposition 3.2

Proposition 3.5 Under the splitting property (20) there is equivalence between (i) $W$ is $\mathbb{G}$-Markov with transition matrix $R=\bar{R}\left(\mathbf{1}_{m}^{\top} \otimes I_{n}\right)$.

(ii) $X$ is $\mathbb{G}$-Markov with a transition matrix $A$.

Moreover, in each of these cases we have the relation $\bar{R}=\left(I_{m} \otimes A\right) \Delta(G)$.

Proof. We omit the proof of proposition 3.5, since it is similar to that of proposition 3.2 .

Remark 3.6 The main message of proposition 3.5 is that to have $W$ Markov with transition probabilities only depending on past values of $X$ it is sufficient to start with a $\mathbb{G}$-Markov chain $X$ and to assume that the splitting property (20) holds. 
Remark 3.7 We noticed in remark 3.4, that from the assumption that $Z$ is $\mathbb{F}$-Markov and the validity of the factorization property, one could deduce that $W$ is $\mathbb{G}$-Markov. Conversely, given that $W$ is $\mathbb{G}-M a r k o v$ with the transition matrix as in (12) above, we can also deduce that $Z$ is $\mathbb{F}$-Markov with $Q$ as in (14) as its transition matrix (and that equation (9) holds). This also follows from more general considerations to be explained at the end of section 4 , but here we give an explicit calculation.

So let $W$ be a $\mathbb{G}$-Markov process with transition matrix $R=\bar{R}\left(\mathbf{1}_{m}^{\top} \otimes I_{n}\right)$. Then $E\left[W_{t} \mid \mathcal{G}_{t-1}\right]=\bar{R} X_{t-1}$. From this it follows that

$$
E\left[X_{t} \mid \mathcal{G}_{t-1}\right]=\left(\mathbf{1}_{m}^{\top} \otimes I_{n}\right) \bar{R} X_{t-1}=A X_{t-1}
$$

with $A=\left(\mathbf{1}_{m}^{\top} \otimes I_{n}\right) \bar{R}$. Furthermore we have $E\left[Y_{t} \mid \mathcal{G}_{t}\right]=\left(I_{m} \otimes \mathbf{1}_{n}^{\top}\right) E\left[W_{t+1} \mid \mathcal{G}_{t}\right]=$ $\left(I_{m} \otimes \mathbf{1}_{n}^{\top}\right) \bar{R} X_{t}=G X_{t}$ with $G=\left(I_{m} \otimes \mathbf{1}_{n}^{\top}\right) \bar{R}$.

We now compute $E\left[Z_{t+1} \mid \mathcal{F}_{t}\right]=E\left[E\left[Y_{t+1} \mid \mathcal{G}_{t+1}\right] \otimes X_{t+1} \mid \mathcal{F}_{t}\right]$. By the relation that we just showed, this becomes $E\left[G X_{t+1} \otimes X_{t+1} \mid \mathcal{F}_{t}\right]$ which is $\Delta(G) E\left[X_{t+1} \mid \mathcal{F}_{t}\right]$. We have reached our goal as soon as we show that $E\left[X_{t+1} \mid \mathcal{F}_{t}\right]=E\left[X_{t+1} \mid \mathcal{G}_{t}\right]$. But it is easy to see that this follows immediately from the splitting property (actually it is equivalent).

Thus we showed the Markov property of $Z$ with respect to $\mathbb{F}$ and found its transition matrix.

Altogether we summarize our findings of this section in

Theorem 3.8 There is equivalence between

(a) $X$ is $\mathbb{F}$-Markov and the factorization property holds.

(b) $X$ is $\mathbb{G}$-Markov and the splitting property holds.

(c) $Z$ is $\mathbb{F}$-Markov with transition matrix $Q$ as in (国).

(d) $W$ is $\mathbb{G}$-Markov with transition matrix $R$ as in $(19)$.

(e) $(X, Y)$ is a hidden Markov chain.

Proof. The equivalence of (a) and (c) is just proposition 3.2, that of (b) and (d) is proposition 3.5. Equivalence of (c) and (d) is the content of remarks 3.4 and 3.7, whereas (c) and (e) are equivalent by definition 2.6 of a hidden Markov chain.

\section{Stochastic systems}

In the previous sections we restricted ourselves to time homogeneous processes, implying that all conditional probabilities and expectations don't depend on time directly. In the present section where explicit calculations are absent, this restriction playes no role. We introduce some notation. Given a stochastic process $\zeta$ with values in some arbitrary measurable space, we denote for all $t$ by $\mathcal{F}_{t}^{\zeta}$ the $\sigma$-algebra generated by the $\zeta_{s}$ for $s \leq t$ and by $\mathcal{F}_{t}^{\zeta+}$ the $\sigma$-algebra generated by the $\zeta_{s}$ for $s \geq t$. Many of the results in the previous sections can be abstractly formulated in terms of properties of stochastic systems. A stochastic system is a formally defined concept. The main ingredients are a state process 
$X$ and an output process $Y$ (defined on a suitable probability space and taking values in some other spaces) and certain conditional independence relations.

Let us therefore recall some facts on conditional independence. Two $\sigma$-algebras $\mathcal{H}_{1}$ and $\mathcal{H}_{2}$ are called conditionally independent given a $\sigma$-algebra $\mathcal{G}$ if for all bounded $\mathcal{H}_{i}$-measurable functions $H_{i}(i=1,2)$ the relation $E\left[H_{1} H_{2} \mid \mathcal{G}\right]=$ $E\left[H_{1} \mid \mathcal{G}\right] E\left[H_{2} \mid \mathcal{G}\right]$ holds. A convenient characterization of this is that $\sigma$-algebras $\mathcal{H}_{1}$ and $\mathcal{H}_{2}$ are conditionally independent given $\sigma$-algebra $\mathcal{G}$ if for all bounded $\mathcal{H}_{1}$-measurable functions $H_{1}$ the relation $E\left[H_{1} \mid \mathcal{G} \vee \mathcal{H}_{2}\right]=E\left[H_{1} \mid \mathcal{G}\right]$ holds.

In the literature one can find two definitions of a stochastic system, that are slightly different. The first one is due to Picci $|5|$, and the essential part of the definition is that for all $t$ the $\sigma$-algebras $\mathcal{F}_{t+1}^{X+} \vee \mathcal{F}_{t+1}^{Y+}$ and $\mathcal{F}_{t}^{X} \vee \mathcal{F}_{t}^{Y}$ are conditionally independent given $\sigma\left(X_{t}\right)$. The other one is due to Van Schuppen [6] in which the conditional independence relation between $\sigma$-algebras becomes: for all $t$ the $\sigma$-algebras $\mathcal{F}_{t}^{X+} \vee \mathcal{F}_{t}^{Y+}$ and $\mathcal{F}_{t-1}^{X} \vee \mathcal{F}_{t-1}^{Y}$ are conditionally independent given $\sigma\left(X_{t}\right)$. Implications of the two different definitions for the filtering problem will be discussed in section 5 .

We will write $(X, Y) \in \Sigma_{P}$ if the pair of processes $(X, Y)$ is a stochastic system according to [5] and $(X, Y) \in \Sigma_{S}$ if it is one in the sense of [6]. Using this notation, we see that $(X, Y) \in \Sigma_{P}$ is equivalent with saying that $Z$ is an $\mathbb{F}$-Markov process with transition probabilities depending on $X$ only, and that $(X, Y) \in \Sigma_{S}$ is equivalent with saying that $W$ is a $\mathbb{G}$-Markov process with transition probabilities depending on $X$ only. Notice that both for a stochastic system $(X, Y)$ either in $\Sigma_{P}$ or in $\Sigma_{S}$ the state process is always Markov relative to its own filtration.

An obvious relation between the different concepts is that $(X, Y) \in \Sigma_{P}$ iff $(X, \sigma Y) \in \Sigma_{S}$, where $\sigma Y$ is the process defined by $\sigma Y_{t}=Y_{t+1}$. Another relation is given in the following

Proposition 4.1 A pair $(X, Y)$ belongs to $\Sigma_{S}$ and the splitting property holds iff it belongs to $\Sigma_{P}$ and the output property (or the factorization property) holds.

Proof. Suppose that $(X, Y) \in \Sigma_{P}$ and that the output property holds. Since $X$ is $\mathbb{F}$-Markov, we have $E\left[X_{t+1} \mid \mathcal{F}_{t}\right]=E\left[X_{t+1} \mid X_{t}\right]$, which is $\mathcal{G}_{t}$ measurable and therefore equal to $E\left[X_{t+1} \mid \mathcal{G}_{t}\right]$, which is equivalent to the splitting property because of the characterization of conditional independence given at the beginning of this section.

Next we show that $(X, Y)$ also belongs to $\Sigma_{S}$. We compute

$$
\begin{aligned}
E\left[W_{t+1} \mid \mathcal{G}_{t}\right] & =E\left[E\left[W_{t+1} \mid \mathcal{F}_{t}\right] \mid \mathcal{G}_{t}\right] \\
& =E\left[Y_{t} \otimes E\left[X_{t+1} \mid \mathcal{F}_{t}\right] \mid \mathcal{G}_{t}\right] \\
& =E\left[Y_{t} \otimes E\left[X_{t+1} \mid X_{t}\right] \mid \mathcal{G}_{t}\right] \\
& =E\left[Y_{t} \mid \mathcal{G}_{t}\right] \otimes E\left[X_{t+1} \mid X_{t}\right]
\end{aligned}
$$

which is $\sigma\left(X_{t}\right)$-measurable, because of the output property.

Conversely, letting $(X, Y) \in \Sigma_{S}$ we automatically get the output property, because $E\left[Y_{t} \mid \mathcal{G}_{t}\right]=\left(I_{n} \otimes \mathbf{1}_{n}^{\top}\right) E\left[W_{t+1} \mid \mathcal{G}_{t}\right]=\left(I_{n} \otimes \mathbf{1}_{n}^{\top}\right) E\left[W_{t+1} \mid X_{t}\right]$ in view of 
$(X, Y) \in \Sigma_{S}$. Assuming the conditional independence relation we obtain the Markov property of $Z$ from

$$
\begin{aligned}
E\left[Z_{t+1} \mid \mathcal{F}_{t}\right] & =E\left[E\left[Z_{t+1} \mid \mathcal{G}_{t+1}\right] \mid \mathcal{F}_{t}\right] \\
& =E\left[E\left[Y_{t+1} \mid \mathcal{G}_{t+1}\right] \otimes X_{t+1} \mid \mathcal{F}_{t}\right] \\
& =E\left[E\left[Y_{t+1} \mid X_{t+1}\right] \otimes X_{t+1} \mid \mathcal{F}_{t}\right] \text { (output property) } \\
& =E\left[E\left[Y_{t+1} \mid X_{t+1}\right] \otimes X_{t+1} \mid \mathcal{G}_{t}\right] \text { (splitting property) } \\
& =E\left[E\left[Y_{t+1} \mid X_{t+1}\right] \otimes X_{t+1} \mid X_{t}\right] \text { (W is } \mathbb{G} \text {-Markov), }
\end{aligned}
$$

which shows that $(X, Y) \in \Sigma_{P}$.

Remark 4.2 Observe that we already encountered a computational form of this proposition in subsections 3.1 and 3.2 .

The connection between systems in $\Sigma_{P}$ and $\Sigma_{S}$ and Hidden Markov chains is described as

Proposition 4.3 A finite valued time homogeneous system belonging both to $\Sigma_{P}$ and to $\Sigma_{S}$ is a Hidden Markov chain and vice versa.

Proof. If $(X, Y)$ is a HMC, then it follows from theorem 3.8 that it belongs to both $\Sigma_{P}$ and $\Sigma_{S}$. The converse statement follows in a similar way from this theorem.

\section{$5 \quad$ Filtering}

In this section we give some filtering and prediction formulas. By the filtering problem for a system $(X, Y)$ belonging to $\Sigma_{P}$ or to $\Sigma_{S}$ we mean the determination for each $t$ of the conditional law of $X_{t}$ given $Y_{0}, \ldots, Y_{t}$. As before, for each $t$ we denote by $\mathcal{F}_{t}^{Y}$ the $\sigma$-algebra generated by $Y_{0}, \ldots, Y_{t}$. Since the state space of $X$ is a set of basis vectors, this conditional law is completely determined by the conditional expectation $E\left[X_{t} \mid \mathcal{F}_{t}^{Y}\right]$. The prediction problem is to determine for each $t$ the conditional law of $X_{t+1}$ given $Y_{0}, \ldots, Y_{t}$, that is completely characterized by the conditional expectations $E\left[X_{t+1} \mid \mathcal{F}_{t}^{Y}\right]$. We will use the notations $E\left[X_{t} \mid \mathcal{F}_{t}^{Y}\right]=\hat{X}_{t}$ and $E\left[X_{t+1} \mid \mathcal{F}_{t}^{Y}\right]=\hat{X}_{t+1 \mid t}$. Similarly we write $E\left[Y_{t+1} \mid \mathcal{F}_{t}^{Y}\right]=\hat{Y}_{t+1 \mid t}$. In addition to the above one wants to have $\hat{X}_{t}$ and $\hat{X}_{t+1 \mid t}$ in recursive form. We shall see below that the recursions for the cases $(X, Y) \in \Sigma_{P}$ and $(X, Y) \in \Sigma_{S}$ are different.

In the book 2 recursive formulae for unnormalized filters are obtained by a measure transformation. Here we undertake a direct approach, that leads to a simple recursive formula for the conditional probabilities itself. The key argument is in all cases provided by lemma A.1.

\subsection{Filter for $\Sigma_{P}$}

In this section we obtain the filter for a system in $\Sigma_{P}$, so we work with a Markov chain $Z_{t}=X_{t} \otimes Y_{t}$ with transition matrix $Q=\bar{Q}\left(\mathbf{1}_{m}^{\top} \otimes I_{n}\right)$. The matrix $\bar{Q}$ we 
can write as

$$
\bar{Q}=\left[\begin{array}{c}
Q_{1} \\
\vdots \\
Q_{m}
\end{array}\right]
$$

with the $Q_{i}$ in $\mathbb{R}^{n \times n}$. No further assumptions on the $Q_{i}$ are made. Observe that the $Q_{i}$ have the interpretation that

$$
Q_{i} X_{t}=E\left[X_{t+1} 1_{\left\{Y_{t+1}=f_{i}\right\}} \mid \mathcal{F}_{t}\right] .
$$

We have the following result (alternatively presented in [5]).

Theorem 5.1 The filter $\hat{X}$ is given by the recursion

$$
\hat{X}_{t}=\left[\begin{array}{lll}
\frac{Q_{1} \hat{X}_{t-1}}{\mathbf{1}_{n}^{\top} Q_{1} \hat{X}_{t-1}} & \cdots & \frac{Q_{m} \hat{X}_{t-1}}{\mathbf{1}_{n}^{\top} Q_{m} \hat{X}_{t-1}}
\end{array}\right] Y_{t}
$$

with the initial condition determined by the initial law of $Z$. The prediction $\hat{X}_{t+1 \mid t}$ is equal to $A \hat{X}_{t}$ with $A=\sum_{i=1}^{m} Q_{i}$ and $X_{0 \mid-1}=E X_{0}=p_{0}$. For the prediction $\hat{Y}_{t+1 \mid t}$ we have $\hat{Y}_{t+1 \mid t}=C \hat{X}_{t}$ with $C=\left(I_{m} \otimes \mathbf{1}_{n}^{\top}\right) \bar{Q}$.

Proof. We use equation (35) with $\mathcal{F}^{0}=\mathcal{F}_{t}^{Y}, \mathcal{H}=\sigma\left(Y_{t+1}\right)$, which is generated by the sets $H_{i}=\left\{Y_{t+1}=f_{i}\right\}$ and $U=X_{t+1}$. Thus we obtain

$$
E\left[X_{t+1} \mid \mathcal{F}_{t+1}^{Y}\right]=\sum_{i=1}^{m} E_{i}\left[X_{t+1} \mid \mathcal{F}_{t}^{Y}\right] 1_{H_{i}}=\sum_{i=1}^{m} \frac{E\left[X_{t+1} 1_{H_{i}} \mid \mathcal{F}_{t}^{Y}\right]}{P\left(H_{i} \mid \mathcal{F}_{t}^{Y}\right)} 1_{H_{i}}
$$

Then we use the Markov property of $Z$ to write

$$
E\left[X_{t+1} 1_{H_{i}} \mid \mathcal{F}_{t}^{Y}\right]=E\left[E\left[X_{t+1} 1_{H_{i}} \mid \mathcal{F}_{t}\right] \mid \mathcal{F}_{t}^{Y}\right]=E\left[Q_{i} X_{t} \mid \mathcal{F}_{t}^{Y}\right]=Q_{i} \hat{X}_{t} .
$$

Since $P\left(H_{i} \mid \mathcal{F}_{t}^{Y}\right)=E\left[1_{H_{i}} \mid \mathcal{F}_{t}\right]=\mathbf{1}_{n}^{\top} E\left[X_{t+1} 1_{H_{i}} \mid \mathcal{F}_{t}^{Y}\right]$ we get equation (23).

Define now $A=\sum_{i=1}^{m} Q_{i}=\left(\mathbf{1}_{m}^{\top} \otimes I_{n}\right) \bar{Q}$ and $C=\left(I_{m} \otimes \mathbf{1}_{n}^{\top}\right) \bar{Q}$. Then we have $E\left[X_{t+1} \mid \mathcal{F}_{t}\right]=A X_{t}$ and $E\left[Y_{t+1} \mid \mathcal{F}_{t}\right]=C X_{t}$. As a consequence we get by reconditioning that $\hat{X}_{t+1 \mid t}=A \hat{X}_{t}$ and that $\hat{Y}_{t+1 \mid t}=C \hat{X}_{t}$.

We see that the filter $\hat{X}_{t}$ satisfies a completely recursive system, that is, $\hat{X}_{t}$ is completely determined by $\hat{X}_{t-1}$ and $Y_{t}$. In absence of further conditions on the matrix $Q$ (in particular the factorization property) there seems to be no complete recursion that is satisfied by $X_{t \mid t-1}$. The reason for this is that we don't have the Markov property of $W$ with respect to $\mathbb{G}$, unless the factorization property holds, in which case the formulas above take a particular nice form. See subsection 5.3 .

Remark 5.2 It follows from equation (22) that the filter (23) can alternatively be expressed as

$$
\hat{X}_{t}=\left[Q_{1} \hat{X}_{t-1}, \ldots, Q_{m} \hat{X}_{t-1}\right] \operatorname{diag}\left(C \hat{X}_{t-1}\right)^{-1} Y_{t} .
$$


Indeed, from equation (22) we obtain $\mathbf{1}^{\top} Q_{i} X_{t}=P\left(Y_{t+1}=f_{i} \mid \mathcal{F}_{t}\right)$, hence $E\left[Y_{t+1} \mid \mathcal{F}_{t}\right]$ is the vector with elements $\mathbf{1}^{\top} Q_{i} X_{t}$. Conditioning of this vector on $\mathcal{F}_{t}^{Y}$ gives that $\hat{Y}_{t+1 \mid t}$ is the vector with elements $\mathbf{1}^{\top} Q_{i} \hat{X}_{t}$. So we can rewrite (23) as $\hat{X}_{t}=\left[Q_{1} \hat{X}_{t-1}, \ldots, Q_{m} \hat{X}_{t-1}\right] \operatorname{diag}\left(Y_{t \mid t-1}\right)^{-1} Y_{t}$ and the result follows.

\subsection{Filter for $\Sigma_{S}$}

In this section we obtain the filter for a system in $\Sigma_{S}$, so we work with a Markov chain $W_{t}=X_{t} \otimes Y_{t-1}$ with transition matrix $R=\bar{R}\left(\mathbf{1}_{m}^{\top} \otimes I_{n}\right)$, where the matrix $\bar{R}$ can be written as

$$
\bar{R}=\left[\begin{array}{c}
R_{1} \\
\vdots \\
R_{m}
\end{array}\right]
$$

for certain matrices $R_{i}$ in $\mathbb{R}^{n \times n}$. No further assumptions on the $R_{i}$ are made. Observe that the $R_{i}$ have the interpretation that

$$
R_{i} X_{t}=E\left[X_{t+1} 1_{\left\{Y_{t}=f_{i}\right\}} \mid \mathcal{G}_{t}\right] .
$$

Then we have

Theorem 5.3 The predictor $\hat{X}_{t \mid t-1}$ is given by the recursion

$$
\hat{X}_{t+1 \mid t}=\left[\begin{array}{lll}
\frac{R_{1} \hat{X}_{t \mid t-1}}{\mathbf{1}_{n}^{\top} R_{1} \hat{X}_{t \mid t-1}} & \cdots & \frac{R_{m} \hat{X}_{t \mid t-1}}{\mathbf{1}_{n}^{\top} R_{m} \hat{X}_{t \mid t-1}}
\end{array}\right] Y_{t}
$$

with the initial condition $X_{0 \mid-1}=E X_{0}$. For the filter $\hat{X}_{t}$ and for $\hat{Y}_{t+1 \mid t}$ we have the following relations.

$$
\hat{X}_{t+1}=\operatorname{diag}\left(\hat{X}_{t+1 \mid t}\right) G^{\top} \operatorname{diag}\left(\hat{Y}_{t+1 \mid t}\right)^{-1} Y_{t+1},
$$

where $G=\left(I_{m} \otimes \mathbf{1}_{n}^{\top}\right) \bar{R}$ and

$$
\hat{Y}_{t+1 \mid t}=G X_{t+1 \mid t} \text {. }
$$

Proof. We use equation (35) with $\mathcal{F}^{0}=\mathcal{F}_{t-1}^{Y}, \mathcal{H}=\sigma\left(Y_{t}\right)$, which is generated by the sets $H_{i}=\left\{Y_{t}=f_{i}\right\}$ and $U=X_{t+1}$. Then we obtain $E\left[X_{t+1} \mid \mathcal{F}_{t}^{Y}\right]=$ $\sum_{i=1}^{m} E_{i}\left[X_{t+1} \mid \mathcal{F}_{t-1}^{Y}\right] 1_{H_{i}}=\sum_{i=1}^{m} \frac{E\left[X_{t+1} 1_{H_{i}} \mid \mathcal{F}_{t-1}^{Y}\right]}{P\left(H_{i} \mid \mathcal{F}_{t}^{Y}\right)} 1_{H_{i}}$. Then we use the Markov property of $W$ to write

$$
\begin{aligned}
E\left[X_{t+1} 1_{H_{i}} \mid \mathcal{F}_{t-1}^{Y}\right] & =E\left[E\left[X_{t+1} 1_{H_{i}} \mid \mathcal{G}_{t}\right] \mid \mathcal{F}_{t-1}^{Y}\right] \\
& =E\left[R_{i} X_{t} \mid \mathcal{F}_{t-1}^{Y}\right] \\
& =R_{i} \hat{X}_{t \mid t-1}
\end{aligned}
$$


Since $P\left(H_{i} \mid \mathcal{F}_{t-1}^{Y}\right)=E\left[1_{H_{i}} \mid \mathcal{F}_{t-1}^{Y}\right]=\mathbf{1}_{n}^{\top} E\left[X_{t+1} 1_{H_{i}} \mid \mathcal{F}_{t-1}^{Y}\right]$ we get equation (26). To derive the formula (27) for the filter we proceed similarly, using lemma A.1 again with $U=X_{t+1}, \mathcal{F}^{0}=\mathcal{F}_{t}^{Y}$ and $\mathcal{H}=\sigma\left(Y_{t+1}\right)$ generated by the sets $H_{i}=\left\{Y_{t+1}=f_{i}\right\}$. Then we can write equation (35) as $E\left[X_{t+1} \mid \mathcal{F}_{t+1}^{Y}\right]=$ $E\left[X_{t+1} Y_{t+1}^{\top} \mid \mathcal{F}_{t}^{Y}\right] \operatorname{diag}\left(\hat{Y}_{t+1 \mid t}\right)^{-1} Y_{t+1}$.

$$
\begin{aligned}
E\left[X_{t+1} Y_{t+1}^{\top} \mid \mathcal{F}_{t}^{Y}\right] & =E\left[E\left[X_{t+1} Y_{t+1}^{\top} \mid \mathcal{G}_{t+1}\right] \mid \mathcal{F}_{t}^{Y}\right] \\
& =E\left[X_{t+1} E\left[Y_{t+1}^{\top} \mid \mathcal{G}_{t+1}\right] \mid \mathcal{F}_{t}^{Y}\right] \\
& =E\left[X_{t+1}\left(G X_{t+1}\right)^{\top} \mid \mathcal{F}_{t}^{Y}\right] \\
& =E\left[\operatorname{diag}\left(X_{t+1}\right) \mid \mathcal{F}_{t}^{Y}\right] G^{\top} .
\end{aligned}
$$

Then equation (27) follows, as well as equation (28), since we have $E\left[Y_{t+1} \mid \mathcal{F}_{t}^{Y}\right]=$ $E\left[Y_{t+1} X_{t+1}^{\top} \mid \mathcal{F}_{t}^{Y}\right] \mathbf{1}_{n}=G \operatorname{diag}\left(\hat{X}_{t+1 \mid t}\right) \mathbf{1}_{n}=G \hat{X}_{t+1 \mid t}$.

Remark 5.4 By a similar argument as in remark 5.2 we can rewrite the recursion (26) for the predictor as

$$
\hat{X}_{t+1 \mid t}=\left[R_{1} \hat{X}_{t \mid t-1}, \ldots, R_{m} \hat{X}_{t \mid t-1}\right] \operatorname{diag}\left(G \hat{X}_{t \mid t-1}\right)^{-1} Y_{t}
$$

Remark 5.5 Notice that in contrast with what we got in subsection 5.1 for $\Sigma_{P}$ here the predictor satisfies a completely recursive system, whereas we obtain the filter in terms of the predictor.

The formulas above take a particular nice form if the system satisfies the splitting property. See subsection 5.3 .

\subsection{Filter for a Hidden Markov Chain}

In this section we return to the setting of sections 2 and 3 and we give the recursive filtering formula for the stochastic system with the HMC $Y$ as its output. Therefore, we can apply the results of subsection 5.1 with the specification that $\bar{Q}=\Delta(G) A$, so we have $Q_{i}=\operatorname{diag}\left(G_{i}\right) A$ and $\mathbf{1}_{n}^{\top} Q_{i}=G_{i .}$. . The following holds.

Theorem 5.6 (i) The conditional distribution of the $X_{t}$ given $Y_{0}, \ldots, Y_{t}$ is recursively determined by

$$
\hat{X}_{t}=\operatorname{diag}\left(A \hat{X}_{t-1}\right) G^{\top} \operatorname{diag}\left(G A \hat{X}_{t-1}\right)^{-1} Y_{t}
$$

with initial condition $\hat{X}_{0}=\operatorname{diag}\left(p_{0}\right) G^{\top} \operatorname{diag}\left(G p_{0}\right)^{-1} Y_{0}$, with $p_{0}=E X_{0}$.

(ii) The conditional distribution of the $X_{t}$ given $Y_{0}, \ldots, Y_{t-1}$ is recursively determined by

$$
\hat{X}_{t+1 \mid t}=\operatorname{Adiag}\left(\hat{X}_{t \mid t-1}\right) G^{\top} \operatorname{diag}\left(G \hat{X}_{t \mid t-1}\right)^{-1} Y_{t}
$$


with initial condition $X_{0 \mid-1}=E X_{0}=p_{0}$.

(iii) The conditional expectation $\hat{Y}_{t+1 \mid t}=E\left[Y_{t+1} \mid \mathcal{F}_{t}^{Y}\right]$ is given by

$$
\hat{Y}_{t+1 \mid t}=G A \operatorname{diag}\left(\hat{X}_{t \mid t-1}\right) G^{\top} \operatorname{diag}\left(G \hat{X}_{t \mid t-1}\right)^{-1} Y_{t}
$$

Proof. (i) Just use equation (23) and notice that

$$
\frac{Q_{i} \hat{X}_{t-1}}{\mathbf{1}_{n}^{\top} Q_{i} \hat{X}_{t-1}}=\operatorname{diag}\left(A \hat{X}_{t-1}\right) \frac{G_{i}^{\top}}{\left(G A \hat{X}_{t-1}\right)_{i}} .
$$

(ii) follows from (i), since we know from theorem 5.1 that $\hat{X}_{t+1 \mid t}=A \hat{X}_{t}$.

(iii) also follows from theorem 5.1, upon noticing that $C$ now becomes $G A$ in view of (5).

Remark 5.7 Here both the filter and the predictor satisfy a complete recursive system. This is not surprising, because a HMC is a stochastic system belonging to both $\Sigma_{P}$ and $\Sigma_{S}$. Notice that theorem 5.6 can alternatively be derived from theorem 5.3, since under the assumptions of the present subsection we have that $R_{i}=\operatorname{Adiag}\left(G_{i}\right)$.

Remark 5.8 If we define for $x \in \mathbb{R}_{+}^{n}$ the matrix

$$
G_{x}:=\operatorname{diag}(x) G^{\top} \operatorname{diag}(G x)^{-1},
$$

then equations (29), (30) and (31) take the form $\hat{X}_{t}=G_{A \hat{X}_{t-1}} Y_{t}, \hat{X}_{t+1 \mid t}=$ $A G_{\hat{X}_{t \mid t-1}} Y_{t}$ and $\hat{Y}_{t+1 \mid t}=G A G_{\hat{X}_{t \mid t-1}} Y_{t}$.

One may check that under the condition that $Y$ is a deterministic function of $X$ (in which case the columns of $G$ are basis vectors of $\mathbb{R}^{m}$ ) the matrices $G_{x}$ are right pseudo-inverses of $G$.

\section{References}

[1] L.E. Baum and T. Petrie (1966), Statistical inference for probabilistic functions of finite state Markov chains, Ann. Math. Stat. 37, pp. 1554- 1563.

[2] R. J. Elliott, L. Aggoun and J. B. Moore (1995), Hidden Markov Models. Estimation and Control, Springer.

[3] L. Finesso (1990), Consistent Estimation of the Order for Markov and Hidden Markov Chains, Dissertation University of Maryland.

[4] J.R. Magnus \& H. Neudecker (1988), Matrix Differential Calculus with Applications in Statistics and Econometrics, Wiley. 
[5] G. Picci (1978), On the internal structure of finite state stochastic processes, in Recent Developments in Variable Structure Systems, Springer Lecture Notes in Economics and Math. systems, Vol. 162.

[6] J.H. van Schuppen (1989), Stochastic realization problems, In Three decades of Mathematical System Theory, H. Nijmeijer, J.M. Schumacher (Eds.), Springer Lecture Notes in Control and Information Sciences 135, pp. $480-523$.

[7] P.J.C. Spreij (2001), On the Markov property of a hidden Markov chain, Statistics and Probability Letters, Vol 52/3, pp 279-288.

\section{A A lemma on conditional expectations}

Consider some probability space $(\Omega, \mathcal{F}, P)$ and let $\mathcal{H}$ be a sub- $\sigma$-algebra of $\mathcal{F}$ that is generated by a finite partition $\left\{H_{1}, \ldots, H_{k}\right\}$ of $\Omega$, satisfying $P\left(H_{i}\right)>0$ for all $i$. We introduce the (conditional) probability measures $P_{i}$ on $(\Omega, \mathcal{F})$ defined by $P_{i}(F)=E\left[1_{F} \frac{1_{H_{i}}}{P\left(H_{i}\right)}\right]=P\left(F \mid H_{i}\right)$. Expectation with respect to $P_{i}$ is denoted by $E_{i}$. Notice that for a random variable $X$ with finite expectation we have

$$
E 1_{H_{i}} X=P\left(H_{i}\right) E_{i} X
$$

We also have that for any sub- $\sigma$-algebra $\mathcal{F}_{0}$ and an integrable random variable $X$ the equality

$$
E\left[X 1_{H_{i}} \mid \mathcal{F}_{0}\right] 1_{H_{i}}=P\left(H_{i} \mid \mathcal{F}_{0}\right) E_{i}\left[X \mid \mathcal{F}_{0}\right] .
$$

Recall that for any integrable random variable $U$ it holds that

$$
E[U \mid \mathcal{H}]=\sum_{i=1}^{k} E_{i}[U] 1_{H_{i}}
$$

We extend this result in the following easy to prove lemma. It is used frequently in sections 3 , 1 and 5 .

Lemma A.1 Let $\mathcal{F}^{0}$ be some sub- $\sigma$-algebra of $\mathcal{F}$. Then the following equalities hold true.

$$
\begin{aligned}
E\left[U \mid \mathcal{F}^{0} \vee \mathcal{H}\right] & =\sum_{i=1}^{k} E_{i}\left[U \mid \mathcal{F}^{0}\right] 1_{H_{i}} . \\
E\left[U \mid \mathcal{F}^{0}\right] & =\sum_{i=1}^{k} E_{i}\left[U \mid \mathcal{F}^{0}\right] E\left[1_{H_{i}} \mid \mathcal{F}^{0}\right] . \\
E\left[1_{H_{j}} U \mid \mathcal{F}^{0}\right] & =E\left[1_{H_{j}} \mid \mathcal{F}^{0}\right] E_{j}\left[U \mid \mathcal{F}^{0}\right] .
\end{aligned}
$$


Proof. Concerning the first equality we have to show that

$$
E\left\{1_{F \cap H_{j}} U\right\}=E\left\{1_{F \cap H_{j}} \sum_{i=1}^{k} E_{i}\left[U \mid \mathcal{F}^{0}\right] 1_{H_{i}}\right\}
$$

for all $F \in \mathcal{F}^{0}$, because every set in $\mathcal{F}^{0} \vee \mathcal{H}$ can be written as a finite union of sets $F \cap H_{j}$ with some $F \in \mathcal{F}^{0}$ and because the RHS of (35) is clearly $\mathcal{F}^{0} \vee \mathcal{H}$-measurable. We develop

$$
\begin{aligned}
E\left\{1_{F \cap H_{j}} \sum_{i=1}^{k} E_{i}\left[U \mid \mathcal{F}^{0}\right] 1_{H_{i}}\right\} & =E\left\{1_{F \cap H_{j}} E_{j}\left[U \mid \mathcal{F}^{0}\right]\right\} \\
& =E_{j}\left\{1_{F} E_{j}\left[U \mid \mathcal{F}^{0}\right]\right\} P\left(H_{j}\right) \\
& =E_{j}\left\{1_{F} U\right\} P\left(H_{j}\right) \\
& =E\left\{1_{F \cap H_{j}} U\right\} .
\end{aligned}
$$

In these computations we used (32) in the second and fourth equality and the defining property of conditional expectation in the third. This proves (35).

The second equality is a direct consequence of the first by conditioning on $\mathcal{F}^{0}$. The third equality follows from the second one by taking $1_{H_{j}} U$ instead of $U$.

Remark A.2 If we take in lemma A.1 $\mathcal{F}^{0}$ the trivial $\sigma$-algebra, then (35) reduces to (34). If $P\left(H_{i}\right)=0$ for some $i$, then $P_{i}$ is not well defined but (35) is still valid provided we define $E_{i}\left[U \mid \mathcal{F}^{0}\right]$ to be zero for such an $i$.

Remark A.3 Equation (37) is also known as the conditional Bayes theorem, cf. [2, page 23]. 